\title{
Discrimination and Genetic Diversity among Cultivated Olives of Greece Using RAPD Markers
}

\author{
N. Nikoloudakis, G. Banilas, F. Gazis, and P. Hatzopoulos ${ }^{1}$ \\ Molecular Biology Laboratory, Agricultural University of Athens, Iera Odos 75, 11855 Athens, Greece \\ J. Metzidakis \\ Institute of Subtropical Plants and Olive Tree, NAGREF, Agrokipio 73100, Chania, Crete, Greece
}

AdDitIONAL INDEX WORDS. diversity, polymorphism, Olea europaea, cultivars

\begin{abstract}
Random amplified polymorphic DNA (RAPD) markers were used to study the genetic diversity and to discriminate among 33 Greek olive (Olea europaea L.) cultivars. Three feral forms from Crete and five foreign cultivars recently introduced into Greece were also included. Nineteen primers were selected which produced 64 reproducible polymorphic bands in the 41 olive genotypes studied, with an average of 3.4 informative markers per primer. The RAPD markers resulted in $\mathbf{1 3 5}$ distinct electrophoretic patterns, with an average of 7.1 patterns per primer. Based on either unique or combined patterns, all genotypes could be identified. Genetic similarities between genotypes were estimated using the Dice similarity index and these indicated that a high degree of diversity exists within the Greek olive germplasm. Using the unweighted pair-group method (UPGMA) most cultivars were clustered into two main groups according to their fruit size or commercial use (table or olive oil). However, poor correlation was detected between clustering of cultivars and their principal area of cultivation. RAPD marker data were subjected to nonmetric multidimentional scaling (NMDS) which produced results similar to those of the UPGMA analysis. The results presented here contribute to a comprehensive understanding of cultivated Greek olive germplasm and provide information that could be important for cultural purposes and breeding programs.
\end{abstract}

Olive (Olea europaea L.) is a woody species found throughout the Mediterranean basin and is an important oil producing crop. Olive cultivation most likely originated from the eastern regions of the Mediterranean sea (Zohary and Spiegel-Roy, 1975) as early as $3000 \mathrm{BC}$ (Connell, 1994). It spread westward, following the arches of the basin, to the southern parts of Europe and northern Africa. In many of these areas, olive cultivation is nowadays of great economic importance, since olive oil is a basic constituent of the diet and is considered a healthy source of fats (Harwood and Yaqoob, 2002; Wahrburg et al., 2002).

Over the centuries, hundreds of olive cultivars were selected for their qualitative and quantitative traits and for their adaptation to various microclimates in different regions of the Mediterranean basin (Bartolini et al., 1993). However, the existence of synonyms and homonyms has resulted in considerable uncertainty about the current status of olive cultivars in many countries (Connell, 1994). In Greece, over 170 cultivar names, mainly toponyms (place names) are in existence, although the number of true cultivars might be much smaller (Balatsouras, 1984; Lychnos, 1949). Some of this uncertainty could be attributed to environmental and genotype interactions since it is known that cultivation conditions may contribute to changes in morphology (Booth and Davies, 1995; Vergari et al., 1996).

Recently, several molecular marker techniques have been applied to Olea species to analyze germplasm variability and to differentiate between cultivars (Angiolillo et al., 1999; Fabbri et al., 1995; Hess et al., 2000). Among them, the random amplified polymorphic DNA(RAPD) marker technique is a relatively simple and efficient method for discriminating between olive cultivars (Hatzopoulos et al., 2002).

The objective of the present study was to discriminate and to evaluate genetic similarities among the most important Greek olive cultivars using RAPD markers. Five cultivars recently

Received for publication 30 Dec. 2002. Accepted for publication 1 May 2003. 1To whom correspondence should be addressed; e-mail phat@aua.gr. introduced to Greece from other Mediterranean countries and three feral accessions were also included. Greece belongs to one of the primeval centers of olive domestication and hence seems to possess high germplasm variability (Zohary and Spiegel-Roy, 1975). Due to the highly diverse climate and soil in different areas of Greece, some cultivars are well adapted to extreme conditions such as low temperature, high salinity or drought. Moreover, some Greek cultivars are sensitive to common olive pathogens whereas others are resistant (Balatsouras, 1984; Lychnos, 1949). Therefore, genotype information of Greek olive cultivars is important for providing material for cultural purposes and breeding programs.

\section{Materials and Methods}

Plant material. Genotypes studied were previously identified by morphological characters (Lychnos, 1949) and are maintained as representative accessions at a germplasm collection at the Institute of Subtropical Plants and Olive, National Agricultural Research Foundation (NAGREF), Chania, Crete. Denominations of genotypes and their geographic origin (principal area of cultivation) are given in Table 1 and Fig. 1. The three feral forms included in the study exhibit great potential for use as rootstocks. One of them is an 'Oleaster' from Crete, whereas the other two are seedlings that resulted from hybridization between 'Oleasters' and cultivars, although their exact parentages are unclear. Young olive leaves, still attached to branches, were wrapped in moist paper for transportation. Samples were frozen in liquid nitrogen and stored at $-80^{\circ} \mathrm{C}$ until DNA extraction.

DNA EXTRACTION AND RAPD ANALYSIS. Genomic DNA was extracted from young olive leaves according to the CTAB method of Murray and Thompson (1980). The purity and quantity of genomic DNA was determined spectrophotometrically (Hitachi U-1100, Tokyo, Japan) and confirmed using $0.8 \%$ agarose (Gibco BRL, Life Technologies, Paisley, Scotland) gel electrophoresis against known concentrations of unrestricted lambda DNA. Only 
UV absorption ratios $\mathrm{A}_{260} / 280=1.8$ to 2.0 were accepted. Following the above extraction method, $\approx 10 \mu \mathrm{g}$ DNA per gram fresh tissue was isolated. From the 21 decamer oligonucleotides (MWG, Ebersberg, Germany) tested, 19 resulted in polymorphisms and were used for RAPD reactions (Table 2). PCR reactions were performed in a total volume of $25 \mu \mathrm{L}$ containing $5 \mathrm{ng}$ of template DNA, $0.4 \mu \mathrm{M}$ of a single decamer primer, $100 \mu \mathrm{M}$ of each dNTP and $0.75 \mathrm{U}$ of DNA polymerase (Expand High Fidelity, Boehringer, Mannheim) in the incubation buffer provided by the manufacturer. Error was minimized by making one large batch (master mix) of all reagents for each primer. Acontrol PCR reaction containing all components except for template DNA was included to check for DNA contamination. Amplification reactions were performed in a PTC-200 Peltier thermal cycler (M.J. Research Inc., Watertown, Mass.), which was programmed as follows: 94
${ }^{\circ} \mathrm{C}$ for $2 \mathrm{~min}$, followed by 46 cycles of $94{ }^{\circ} \mathrm{C}$ for $45 \mathrm{~s}, 36{ }^{\circ} \mathrm{C}$ for $1 \mathrm{~min}, 72^{\circ} \mathrm{C}$ for $2 \mathrm{~min}$, and a final extension of $72^{\circ} \mathrm{C}$ for $5 \mathrm{~min}$. Amplified products were separated by gel electrophoresis in $1.2 \%$ agarose and TAE buffer (0.04 M Tris-acetate, $0.001 \mathrm{M}$ EDTA, $\mathrm{pH}$ 8.0). PCR products were detected by ethidium bromide staining and photographed under UV light, with a Gel Doc 1000 camera (Molecular Analyst program of Bio-Rad, Hercules, Calif.). Molecular weights were estimated using the X174/HaeIII marker (F-302SD, Finnzymes, Espoo, Finland).

DATA ANALYSIS. Each gel was analyzed by scoring the presence (1) or absence (0) of polymorphic bands in individual lanes. RAPD fingerprints were scored extremely stringently. Only bands consistently scored from three different PCR reactions were used for the subsequent analysis. The NTSYS-pc software, version $2.02 \mathrm{i}$, was used to estimate genetic similarities with the Dice

Table 1. List of olive genotypes studied, their origin or principal area of cultivation, their fruit size and commercial use.

\begin{tabular}{|c|c|c|c|}
\hline Genotype & Code & Origin-principal area of cultivation ${ }^{z}$ & Fruit sizey/use ${ }^{x}$ \\
\hline Amygdalolia & 33 & Gr-Fokida, Sterea Hellas (SH) & $\mathrm{L} / \mathrm{T}$ \\
\hline Amygdalolia semidwarf & 34 & Gr-Chania, Crete (C) & $\mathrm{L} / \mathrm{T}$ \\
\hline Aggouromanakolia & 21 & Gr-Argolida, Peloponnese (P) & $\mathrm{M} / \mathrm{O}$ \\
\hline Blanquete des Elves & 37 & Portugal (Port) & $\mathrm{L} / \mathrm{O}+\mathrm{T}$ \\
\hline Chrysolia & 31 & Gr-Attiki, Sterea Hellas (SH) & $\mathrm{S} / \mathrm{O}$ \\
\hline Dafnelia & 19 & Gr-Samos, Aegean islands (A) & $\mathrm{M} / \mathrm{O}$ \\
\hline Frantoio & 18 & Italy (It) & $\mathrm{M} / \mathrm{O}$ \\
\hline Frantoio Rhodes & 13 & Gr-Rhodes, Aegean islands (A) & $\mathrm{S} / \mathrm{O}$ \\
\hline Gaidourelia & 28 & Gr-Thiva, Sterea Hellas (SH) & $\mathrm{M} / \mathrm{T}$ \\
\hline Karydolia & 12 & Gr-Chalkidiki, Macedonia (Ma) & $\mathrm{L} / \mathrm{T}$ \\
\hline Kolympada & 15 & Gr-Attiki, Sterea Hellas (SH) & $\mathrm{M} / \mathrm{O}+\mathrm{T}$ \\
\hline Koroneiki & 10 & Gr- Koroni, Peloponnese (P) & $\mathrm{S} / \mathrm{O}$ \\
\hline Kothreiki & 30 & Gr-Fokida, Sterea Hellas (SH) & $\mathrm{M} / \mathrm{O}+\mathrm{T}$ \\
\hline Koutsourelia & 25 & Gr-Lakonia, Peloponnese (P) & $\mathrm{S} / \mathrm{O}$ \\
\hline Leccino & 22 & Italy (It) & $\mathrm{M} / \mathrm{O}$ \\
\hline Lianolia & 4 & Gr-Corfu, Ionian islands (I) & $\mathrm{S} / \mathrm{O}$ \\
\hline Manzanilla & 40 & Spain $(\mathrm{Sp})$ & $\mathrm{L} / \mathrm{T}$ \\
\hline Mastoidis & 2 & Gr-Chania, Crete $(\mathrm{C})$ & $\mathrm{M} / \mathrm{O}$ \\
\hline Mavrelia & 16 & Gr-Lefkada, Ionian islands (I) & $\mathrm{S} / \mathrm{O}$ \\
\hline Megaritiki & 9 & Gr-Attiki, Sterea Hellas (SH) & $\mathrm{M} / \mathrm{O}+\mathrm{T}$ \\
\hline Myrtolia & 29 & Gr-Corfu, Ionian islands (I) & $\mathrm{M} / \mathrm{O}$ \\
\hline Throumpolia & 8 & Gr-Rethymno, Crete (C) & $\mathrm{M} / \mathrm{O}$ \\
\hline Tragolia & 24 & Gr-Kefalonia, Ionian islands (I) & $\mathrm{S} / \mathrm{O}$ \\
\hline Valanolia & 7 & Gr-Lesvos, Aegean islands (A) & $\mathrm{M} / \mathrm{O}$ \\
\hline Vasilikada & 32 & Gr-Corfu, Ionian islands (I) & $\mathrm{L} / \mathrm{T}$ \\
\hline
\end{tabular}

${ }^{2} \mathrm{Gr}=$ Greece.

$\mathrm{y} \mathrm{L}=$ large $; \mathrm{M}=$ medium; $\mathrm{S}=$ small.

× $\mathrm{T}=$ table $; \mathrm{O}=$ oil $; \mathrm{O}+\mathrm{T}=$ both uses $; \mathrm{R}=$ rootstock . 
coefficient (Dice, 1945). The matrix of similarities generated was analyzed by the unweighted pair-group method with arithmetic averages (UPGMA), using the SAHN clustering module. The COPH module was applied to compute a cophenetic value matrix using the UPGMA matrix. The MXCOMP module was then used to compute the cophenetic correlation, i.e., to test the goodness of fit of the cluster analysis to the similarity matrix. Clustering of genotypes was further evaluated by the analysis of low frequency bands (LFBs) (Bourquin et al., 1993), i.e., bands that were shared by only $5(12.2 \%)$ or less genotypes from the 41 studied. The MXCOMP module was used to evaluate the correlation between the original similarity matrix and the similarity matrix after discarding LFBs. COPH and MXCOMP modules

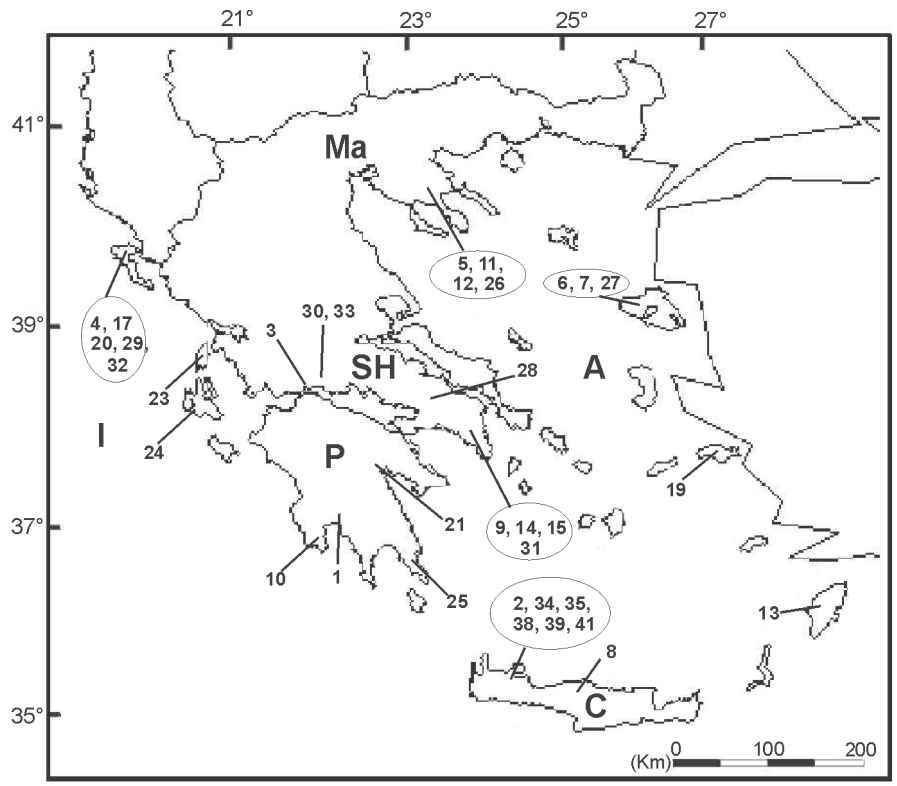

Fig. 1. Map of Greece indicating the origin or principal area of cultivation of olive accessions studied. The numbers refer to the codes of genotypes as shown in Table 1. $\mathrm{A}=$ Aegean islands, $\mathrm{C}=$ Crete, $\mathrm{I}=$ Ionian islands, $\mathrm{Ma}=$ Macedonia, $\mathrm{P}=$ Peloponnese, $\mathrm{SH}=$ Sterea Hellas. were also used to compare the original UPGMA dendrogram with the dendrogram resulted after discarding LFBs. The matrix of similarities was then analyzed using nonmetric multidimensional scaling (NMDS). NMDS was performed 30 times from an initial random configuration, using the MD Scale module of the NTSYS-pc software. A further iteration was performed using the resemblance matrix from principal coordinated analysis (PCOORDA) as an initial configuration. The fit of the NMDS rendering to the original matrix of similarities was measured by Stress coefficient between the two, with Stress approaching zero when the fit is perfect (Kruskal, 1964a, 1964b).

\section{Results}

RAPD PROFILES. In preliminary experiments of RAPD reproducibility, it was found that the concentration of template DNA was crucial to obtain the maximum number of reproducible bands. Varying the concentration of template DNA from 5 to 50 $\mathrm{ng}$ revealed that $5 \mathrm{ng}$ resulted a maximum number of reproducible bands, and therefore $5 \mathrm{ng}$ was used in all subsequent PCR reactions.

The RAPD profiles of the 41 genotypes studied (Table 1) using 19 random primers (Table 2) produced a total of 183 bands. However, only 64 polymorphic bands (35\%), ranging from $0.4 \mathrm{~kb}$ to $2.2 \mathrm{~kb}$, were selected as informative RAPD markers since they were easily scorable and reproducible. The lowest reproducibility was found for the largest RAPD bands, and this agrees with other reports (Ellsworth et al., 1993; Vidal et al., 1999b).

On average, each primer produced 3.4 informative markers. Overall, the polymorphic markers yielded 135 different RAPD electrophoretic patterns with an average of 7.1 different patterns per primer. Primer RP-04 showed the highest level of polymorphism (7 informative bands) and was the most discriminating primer producing 18 different RAPD patterns (Table 2). Although a single primer was not adequate to discriminate between all genotypes, either unique or combined patterns could be used to identify all 41 genotypes. Ten low-frequency bands (LFBs) were detected (Table 2), with PR-04 yielding the highest number (3).

Table 2. List of the informative primers selected and the degree of polymorphism obtained among the 41 olive genotypes studied.

\begin{tabular}{|c|c|c|c|c|c|}
\hline No & $\begin{array}{l}\text { Primer } \\
\text { code }\end{array}$ & $\begin{array}{l}\text { Sequences } \\
5^{\prime} \text { to } 3^{\prime}\end{array}$ & $\begin{array}{l}\text { Polymorphic } \\
\text { bands (no.) }\end{array}$ & $\begin{array}{l}\text { Low-frequency } \\
\text { bands (LFBs) }\end{array}$ & $\begin{array}{l}\text { Electrophoretic } \\
\text { patterns }\end{array}$ \\
\hline 1 & RP-01 & TCC GCA ACC A & 2 & -- & 4 \\
\hline 2 & RP-02 & CAC TTC CGC T & 5 & 1 & 11 \\
\hline 3 & RP-03 & AGA ACC GAG G & 4 & 1 & 7 \\
\hline 4 & RP-04 & TCC GCT GAG A & 7 & 3 & 18 \\
\hline 5 & RP-05 & TCC AAC GGC T & 1 & --- & 2 \\
\hline 6 & RP-06 & CAA GGT GGG T & 5 & 3 & 10 \\
\hline 7 & RP-07 & CAG TGG GGA G & 6 & 1 & 10 \\
\hline 8 & RP-08 & GGG CTA GTC A & 1 & --- & 2 \\
\hline 9 & RP-09 & GGC AGT TCT C & 1 & -- & 2 \\
\hline 10 & RP-10 & GTC GTA GCG G & 1 & --- & 2 \\
\hline 11 & RP-11 & AAG CCC CCC A & 6 & 1 & 16 \\
\hline 12 & RP-12 & ACG GCG ATG A & 5 & --- & 15 \\
\hline 13 & RP-14 & ACG CTG CGA C & 2 & --- & 3 \\
\hline 14 & RP-15 & TGG TGC ACT C & 2 & --- & 4 \\
\hline 15 & RP-16 & GAC ACA GCC C & 3 & --- & 6 \\
\hline 16 & RP-17 & AAG GCA CGA G & 4 & --- & 7 \\
\hline 17 & RP-18 & GAA ACG GGT G & 3 & --- & 6 \\
\hline 18 & RP-19 & GGG TAA CGC C & 3 & --- & 5 \\
\hline 19 & RP-21 & CAG CAC CCA C & 3 & --- & 5 \\
\hline Total & & & 64 & 10 & 135 \\
\hline
\end{tabular}




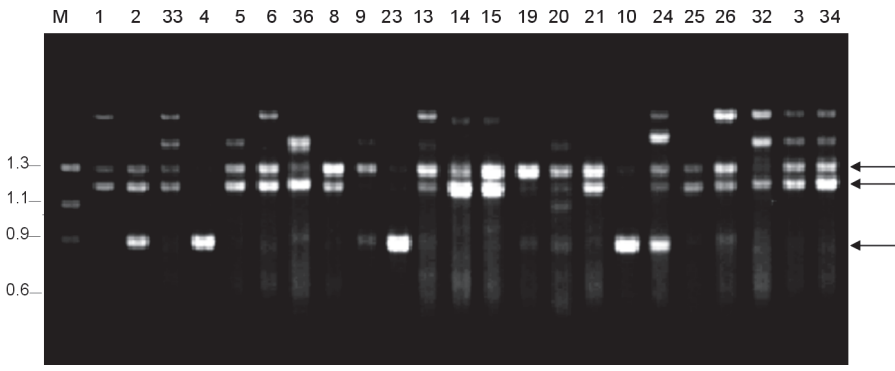

Fig. 2. Examples of DNA polymorphisms detected among olive accessions using primer RP-21. Numbers correspond to accessions in Table 1. M points the molecular size markers. Arrows indicate RAPD bands selected for the analysis.

Using primer RP-03, one specific marker $(\approx 750 \mathrm{bp})$ was detected for 'Frantoio'. Figure 2 shows a representative (of intermediate polymorphism) RAPD profile of 23 accessions using the primer RP-21.

Genetic DIVERSITY. According to the UPGMA dendrogram, based on the Dice coefficient, the cultivars were clustered into two main groups (Fig. 3). The cophenetic correlation revealed a high degree of fit $(r=0.92)$ for the cluster analysis. Group A included 11 accessions, eight $(73 \%)$ of which have large sized fruits and are used as table olives. Within this group, and for a similarity coefficient higher than 0.58 , two subgroups (A1 and A2) were observed. Subgroup A1 was composed of 'Kalamon', 'Chalkidikis', 'Amfissis', and 'Picrolia', four cultivars of different geographical origin. The first three accessions are among the most important Greek table cultivars, whereas the latter is typically used for oil. 'Kalamon' showed the highest similarity value (0.77) to 'Chalkidikis'. Subgroup A2 included three table cultivars from the western Mediterranean Basin (two Spanish and one Portuguese), two Greek table cultivars, 'Amygdalolia' and 'Amygdalolia semidwarf', showing the highest similarity (0.95) and two Greek feral accessions, 'Seedling P2' and 'Oleaster'. Three out of seven Cretan accessions studied clustered in this subgroup. Excluding the last two feral forms, $89 \%$ of accessions within group A are used as table olive cultivars.

Group B contained 23 cultivars, all of Greek origin. Thirteen of them have either small or medium sized fruits and are used for oil, five have either medium or large sized fruits and are typical table olives, and five have medium sized fruits and have dual uses. For a similarity coefficient higher than 0.55 , group B could be divided into three subgroups (B1, B2, and B3). Subgroup B1 consisted of 'Amygdalolia dwarf', a table olive, and 'Mastoidis' that has dual uses (oil and table), both Cretan cultivars. Subgroup B2 included 18 cultivars, 11 that are oil producing cultivars, four that are used for dual use, and three that are table cultivars, 'Chondrolia', 'Karydolia' and 'Gaidourelia'. Within this subgroup, cultivars were further clustered according to their fruit size. 'Koroneiki', 'Thiaki', 'Frantoio Rhodes' and 'Tragolia', all cultivars with small sized fruits, were differentiated from the remaining cultivars of the subgroup B2, the majority of which have medium sized fruits. Within subgroup B2, the highest similarity values were observed between 'Stroggylolia' and 'Kothreiki' (0.86), and between the couples of 'Dafnelia' with each of 'Kolympada'

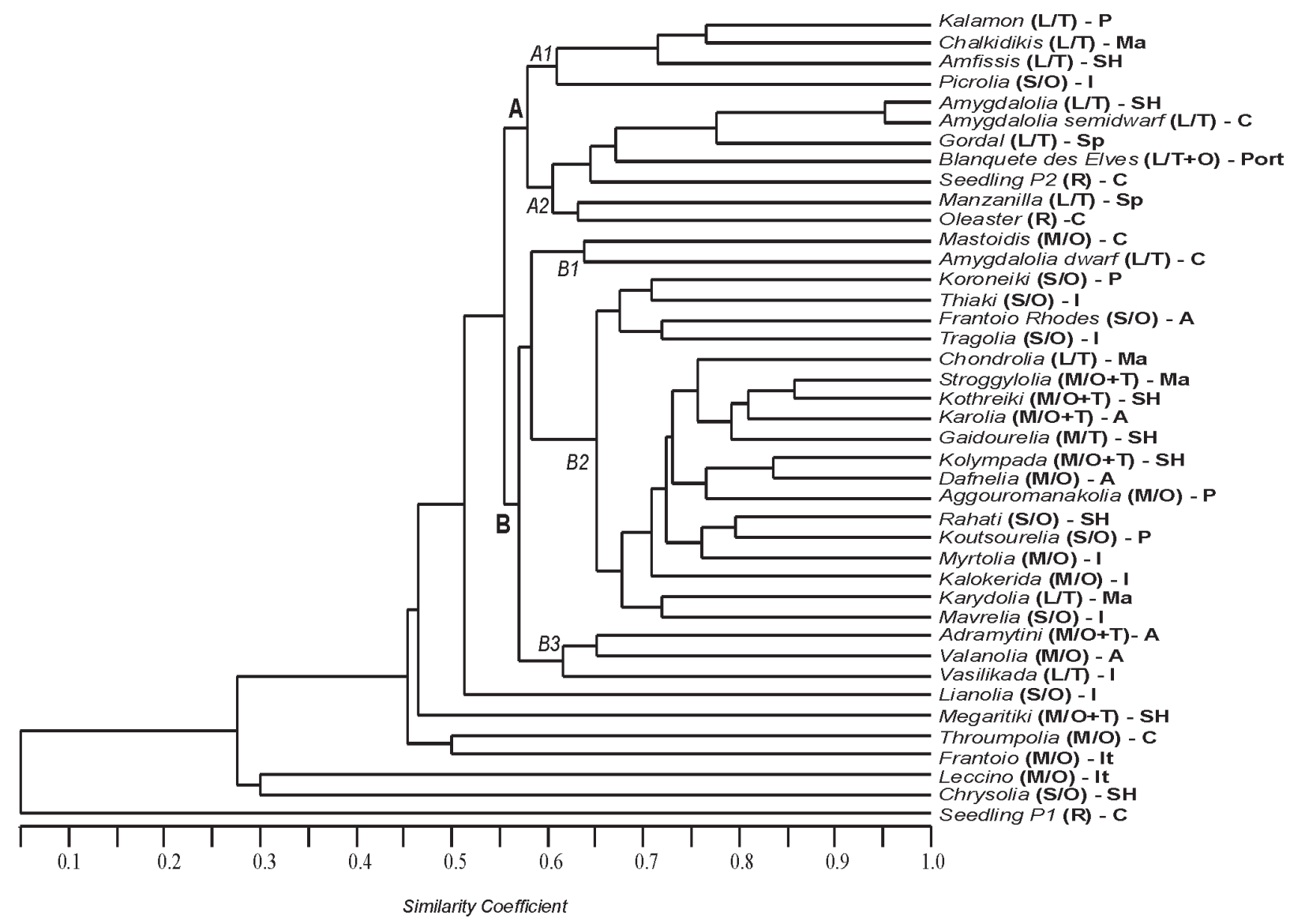

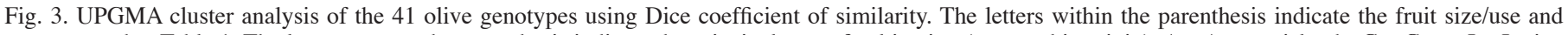
correspond to Table 1 . The letters next to the parenthesis indicate the principal area of cultivation (geographic origin): $\mathrm{A}=\mathrm{Aegean}$ islands, $\mathrm{C}=\mathrm{Crete}, \mathrm{I}=\mathrm{Ionian}$ islands, $\mathrm{It}=$ Italy, $\mathrm{Ma}=$ Macedonia, $\mathrm{P}=$ Peloponnese, $\mathrm{SH}=$ Sterea Hellas, $\mathrm{Sp}=$ Spain. 


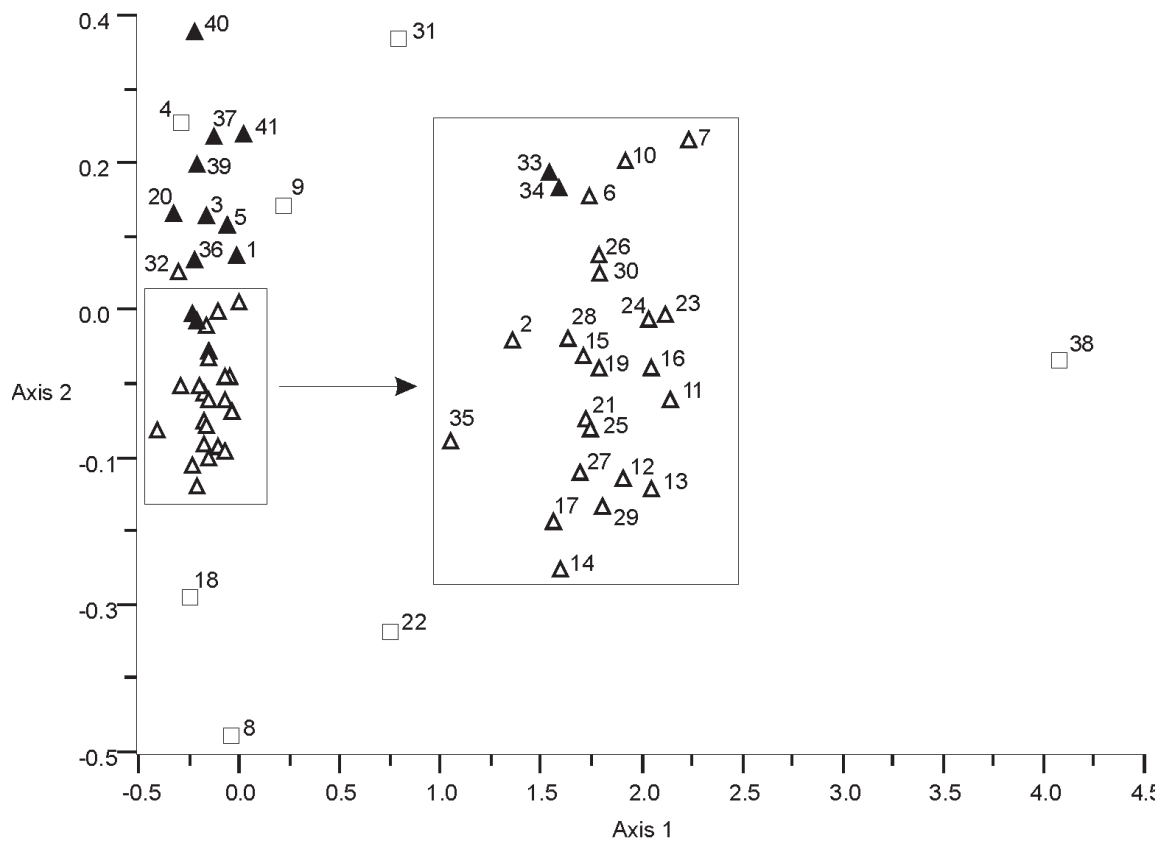

Fig. 4. Results from ordination analysis using non-metric multidimensional scaling (NMDS). Closed triangles represent olive genotypes of group A, open triangles represent group B, and squares represent unrelated genotypes. The numbers refer to the codes of genotypes as shown in Table 1 .

(0.84), 'Aggouromanakolia' (0.82) and 'Gaidourelia' (0.82). The small-sized-fruit 'Mavrelia' showed a genetic similarity of 0.72 to 'Karydolia', a large sized fruit olive. No clear structure of geographic origin could be observed in subgroup B2. However, some cultivars originating from neighboring areas, such as Sterea Hellas, Peloponnese, and Ionian islands (Fig. 1) were clustered together. For instance, 'Koroneiki' and 'Thiaki', and the cluster of 'Rahati', 'Koutsourelia' and 'Myrtolia' (Fig. 3). The third subgroup (B3) consisted of three cultivars, 'Adramytini', 'Valanolia' and 'Vasilikada', the first two of which have medium sized fruits and are of the same origin (Lesvos island), whereas the latter has large sized fruits and originates from a completely different geographic part of Greece (Corfu island) (Fig. 1).

The remaining seven cultivars showed low genetic similarities to each other and to those in groups A and B. Two of them ('Frantoio' and 'Leccino') have Italian origin, one is a feral ('Seedling P1') and the last four are among the most ancient Greek olive cultivars. 'Seedling P1' showed a genetic similarity of only $28 \%$ to all of the other genotypes tested.

LFBs have been reported to contribute to the differentiation of genotypes on a dendrogram (Vidal et al., 1999a; 1999b). No correlation was found between genotypes sharing LFBs and the same genotypes clustering in the UPGMA dendrogram, nor was there any association with geographic origin. Therefore, LFBs did not contribute to the differentiation of genotypes in this study.

NMDS of the genetic similarity matrix produced a two-dimensional plot with a minimum Stress value of $14.5 \%$ deviation between the final NMDS result and the original similarity matrix. When the results of PCOORDA were used as an initial configuration, a better value of Stress $(13.7 \%)$ was obtained, so that the latter configuration is presented here (Fig. 4).

The pattern shown in Fig. 4 was comparable to the clustering observed in the UPGMA dendrogram (Fig. 3). The genotypes of group B were clustered separated from the genotypes of group A, with the exception of 'Vasilikada' (code 32) that has large sized fruits and is a typical table cultivar. As previously observed, the remaining genotypes were shown to be unrelated and were dispersed rather far from each other and from the two previous groups, with the exception of 'Lianolia' (code 4) that was clustered close to either of the two groups in the dendrogram (Fig. 3). Among these accessions, 'Chrysolia' (code 31), one of the most ancient Greek olive cultivar that is also used as an ornamental, and 'Seedling P1' (code 38) were the most isolated genotypes included in this study (Fig. 4).

\section{Discussion}

In the present attempt to discriminate most of the Greek olive cultivars using RAPD markers, a high degree of polymorphism was revealed indicating a wide and diverse genetic background of cultivated olive germplasm in 5 Greece. The results support a report by Loukas and Krimbas (1983) based on allozyme polymorphisms in 22 Greek olive cultivars. Recently, Besnard et al. (2001) studied 96 olive cultivars from the Mediterranean basin using RAPD markers, and found no particular clustering among six Greek cultivars.

The optimum number of primers needed to discriminate among different genotypes depends on the level of genetic variability (Tessier et al., 1999). Mekuria et al. (1999) found that six RAPD primers were adequate to distinguish 39 olive accessions, and Wiesman et al. (1998) used 14 RAPD primers to discriminate eight olive variants of the 'Nabali' cultivar. In the present study, 19 RAPD primers that yielded 64 polymorphic bands were adequate to unequivocally discriminate all 41 genotypes. 'Amygdalolia' and 'Amygdalolia semidwarf' showed the highest degree of similarity (0.95) among all accessions studied. According to Lychnos (1949), 'Amygdololia' is a typical table cultivar from Sterea Hellas (Fig. 1). Therefore, the high genetic similarity between these two genotypes could suggest that 'Amygdalolia semidwarf' was introduced to Crete. On the contrary, 'Amygdalolia dwarf' showed a low similarity $(\approx 0.55)$ to the previous two accessions and therefore, a mislabeling might be possible.

'Oleaster' (code 41) showed the highest genetic similarity (0.64) to 'Manzanilla' (code 40), while in the NMDS plot located close to 'Blanquete des Elves' (code 37) and to 'Seedling P2' (code 39), all clustered in subgroup A2. Although wild olives and cultivars are generally genetically distant, clustering of 'Oleasters' together with certain cultivars has been observed previously (Angiolillo et al., 1999; Bronzini de Caraffa et al., 2002). This could be attributed to the high degree of variability within 'Oleasters' that overlaps the fairly narrow variance expected within cultivars (Lumaret and Ouazzani, 2001).

Most Greek cultivars did not show any particular clustering based on their principal area of cultivation (Figs. 1 and 3). These results could be explained by the fact that some important genotypes were introduced previously to other geographic areas (Lychnos, 1949). However, geographic correlation was observed in few cases, such as for the Cretan accessions in subgroup A2 and subgroup B1, and the cluster of 'Adramytini' and 'Valanolia', two accessions which are among few Greek cultivars that are still being cultivated in a restrictive area (Lesvos island) since ancient years (Balatsouras, 1984). 
The results of this study showed that accessions that clustered together, often shared a use as either table or oil olives or produced similar sized fruits. Genetic differentiation based on olive size and usage has been observed in previous studies (Besnard et al., 2001; Claros et al., 2000; Fabbri et al., 1995). Loukas and Krimbas (1983) studied 22 Greek olive cultivars (21 of which were included in the present study) based on allozyme markers and found clustering according to fruit size rather than to geographic origin.

Wild olives and feral forms are characterized by very small fruits. The correlation between electrophoretic profiles and the common utilization of fruits (oil, table, or dual uses) may be due to either a common origin of cultivars with large sized fruits or to their less affinity to wild olives due to a strong and/or long selection pressure towards larger fruit size (Besnard et al., 2001). Although the sample of foreign table cultivars included in the present study is rather small to extract definite conclusions, taking into account that olive selection has probably occurred in different genetic pools, i.e., Eastern-Western Mediterranean (Besnard et al., 2001), the clustering of the Western table olive cultivars together with the Greek table olives in group A supports the hypothesis for directed clonal selection towards similar agronomic traits. The two Italian oil producing olives, 'Frantoio' and 'Leccino', and the most ancient Greek olive cultivars, especially 'Chrysolia', showed high values of genetic dissimilarity to the currently cultivated germplasm in Greece. Finally, although the denomination 'Frantoio Rhodes' could suggest a close link to the Italian 'Frantoio', such a genetic similarity was not supported by the present data. Thus, as it has been shown previously in the case of olive (Bronzini de Caraffa et al., 2002), a similar name is not a proof of any genetic similarity.

The pattern shown in the NMDS biplot was comparable to the clustering of accessions observed in the dendrogram. The olive cultivars in the NMDS plot could also be clarified into two groups according to fruit size or use.

In conclusion, a high degree of genetic variability was observed among the major Greek olive cultivars studied using RAPD markers. Based on different RAPD patterns, all accessions were identified. Cultivars were generally clustered according to their fruit size or commercial use, while poor correlation was detected between clustering of Greek cultivars and their principal area of cultivation. The effectiveness of the RAPD markers in discriminating the most important Greek olive cultivars, as demonstrated here, could be exploited for screening possible synonyms or homonyms that probably exist within the Greek olive cultivated germplasm, and provide information that could be important for cultural purposes and breeding programs.

\section{Literature Cited}

Angiolillo, A., M. Mencuccini, and L. Baldoni. 1999. Olive genetic diversity assessed using amplified fragment length polymorphisms. Theor. Appl. Genet. 98:411-421.

Balatsouras, G. D. 1984. The olive tree (in Greek). vol.1. Athens, Greece (private publication).

Bartolini, G., C. Messeri, and G. Prevost. 1993. Olive tree germplasm: Descriptor list of cultivated varieties in the world. Acta Hort. 356: 116-118.

Besnard, G., P. Baradat, and A. Bervill. 2001. Genetic relationships in the olive (Olea europaea L.) reflect multilocal selection of cultivars. Theor. Appl. Genet. 102:251-258.

Booth, N. and G. Davies. 1995. An introduction to olive growing in South Australia. Primary industries South Australia and Olive South
Australia, Adelaide, Australia.

Bourquin, J.C., A. Sonko, L. Otten, and B. Walter. 1993. Restriction fragment length polymorphism and molecular taxonomy in Vitis vinifera L. Theor. Appl. Genet. 87:431-438.

Bronzini de Caraffa, V., J. Giannettini, C. Gambotti, and J. Maury. 2002. Genetic relationships between cultivated and wild olives of Corsica and Sardinia using RAPD markers. Euphytica 123:263-271.

Claros, G.M., R. Crespillo, M.L. Aguilar, and F.M. Canovas. 2000. DNA fingerprinting and classification of geographically related genotypes of olive-tree (Olea europaea L.) Euphytica 116:131-142.

Connell, J.H. 1994. History and scope of the olive industry, p. 1-9. In: L. Ferguson, G.S. Sibbett and G.C. Martin (eds). Olive production manual. Univ. California, Division Agricultural and Natural Resources, Oakland, Calif.

Dice, L.R. 1945. Measures of the amount of ecological association between species. Ecology 26:297-302.

Ellsworth, D.L., K.D. Rittenhouse, and R.L. Honeycutt. 1993. Artifactual variation in randomly amplified polymorphic DNA banding patterns. BioTechniques 14:214-217.

Fabbri, A., J.I. Hormaza, and V.S. Polito. 1995. Random amplified polymorphic DNA analysis of olive (Olea europaea L.) cultivars. J. Amer. Soc. Hort. Sci. 120:538-542.

Harwood, J.L. and P. Yaqoob. 2002. Nutritional and health aspects of olive oil. Eur. J. Lipid Sci. Technol. 104: 685-697.

Hatzopoulos, P., G. Banilas, K. Giannoulia, F. Gazis, N. Nikoloudakis, D. Milioni, and K. Haralampidis. 2002. Breeding, molecular markers and molecular biology of the olive tree. Eur. J. Lipid Sci. Technol. 104:574-586.

Hess, J., J.W. Kadareit, and P. Vargas. 2000. The colonization history of Olea europaea L. in Macaronesia based on internal transcribed spacer 1 (ITS-1) sequences, randomly amplified polymorphic DNA(RAPD), and intersimple sequence repeats (ISSR). Mol. Ecol. 9:857-868.

Kruskal, J. B. 1964a. Multidimensional scaling by optimizing goodness of fit to a nonmetric hypothesis. Psychometrika 29:1-27.

Kruskal, J. B. 1964b. Nonmetric multidimensional scaling: A numerical method. Psychometrika 29:28-42.

Loukas, M. and C.B. Krimbas. 1983. History of olive cultivars based on their genetic distances. J. Hort. Sci. 58:121-127.

Lumaret, R. and N. Ouazzani. 2001. Ancient wild olives in Mediterranean forests. Nature 413:700.

Lychnos, N.D. 1949. Olive tree and its cultivation (in Greek). Pyrshos Publ., Athens, Greece.

Mekuria, G.T., G.G. Collins, and M. Sedgley. 1999. Genetic variability between different accessions of some common commercial olive cultivars. J. Hort. Sci. Biotechnol. 74:309-314.

Murray, M.G. and W.F. Thompson. 1980. Rapid isolation of high molecular weight plant DNA. Nucl. Acid Res. 8:4321-4325.

Tessier, C., J. David, P. This, J.M. Boursiquot, and A. Charrier. 1999. Optimization of the choice of molecular markers for varietal identification in Vitis vinifera L. Theor. Appl. Genet. 98:171-177.

Vergari, G., N. Patumi, S. Lavee, and G. Fontanazza 1996. Use of RAPD markers in the characterization of olive germplasm. Olivae 60:19-22.

Vidal, J.R., M. Coarer, and A. Defontaine. 1999a. Genetic relationships among grapevine varieties grown in different French and Spanish regions based on RAPD markers. Euphytica 109:161-172.

Vidal, J.R., S. Moreno, Y. Gogorcena, A. Masa, and J.M. Ortiz. 1999b. On the genetic relationships and origins of six grape cultivars of Galicia (Spain) using RAPD markers. Amer. J. Enol. Viticult. 50:69-75.

Wahrburg, U., M. Kratz, and P. Cullen. 2002. Mediterranean diet, olive oil and health. Eur. J. Lipid Sci. Technol. 104:698-705.

Wiesman, Z., N. Avidan, S. Lavee, and B. Quebedeaux. 1998. Molecular characterization of common olive varieties in Israel and the West Bank using randomly amplified polymorphic DNA (RAPD) markers. J. Amer. Soc. Hort. Sci. 123:837-841.

Zohary, D. and P. Spiegel-Roy. 1975. Beginnings of fruit growing in the old world. Science 187:319-327. 